\title{
banco central de Costa Rica
}

FRANZ SAUTER, ingeniero civil

Profesor de Estructuras Superiores, Universidad de Costa Rica

\section{Sinopsis}

El nuevo edificio, destinado a agrupar los servicios del Banco Central de Costa Rica, está constituido por una estructura de hormigón pretensado. El inmueble dispone de un sótano, estacionamiento propio para 105 vehículos y nueve plantas, con una superficie total de 12.000 metros cuadrados.

Se asienta sobre una base de $40 \times 60 \mathrm{~m}$, donde se levanta un núcleo central de $22 \times 45$ metros.

Está situado en un barrio de calles estrechas, pero tiene su fachada principal frente a una zona verde que le proporcionará mayor categoría y visibilidad.

La estructura se compone de una retícula de vigas pretensadas y montantes. Las vigas se han hormigonado en obra, y las viguetas, también pretensadas, se han prefabricado en taller. El reticulado que en cada planta forman dichos elementos resistentes constituyen el armazón de una losa. Esta forma monolítica da gran rigidez a la estructura.

Las vigas principales, continuas, salvan tramos de $11,25 \mathrm{~m}$ de luz, con secciones varias, $y$ las armaduras de pretensado son de seis cables tipo Leoba, original del Dr. Ing. Fritz Leonhardt, dispuestos en tres capas horizontales, y presentando un perfil parabólico. tensión de tesado fue de $108 \mathrm{~kg} / \mathrm{mm}^{2}$ y el esfuerzo total de pretensado por cable, de $29.700 \mathrm{~kg}$. Los cables se colocaron en el interior de vainas de metal corrugado, las cuales, a su vez, se mantuvieron en posición mediante elementos espaciadores metálicos.

Este edificio se proyectó con fines administrativos para alojar las oficinas y dependencias del Banco Central de Costa Rica, institución encargada de velar por la política monetaria y económica del país. Consta de un sótano, incluyendo área de estacionamiento para 105 vehículos y bóveda de seguridad, y de nueve plantas con una superficie total aprovechable para oficinas y atención del público de 12.000 metros cuadrados. La planta baja y el entresuelo forman un cuerpo rectangular, de $40 \times 60 \mathrm{~m}$, sobre el cual se alza la torre principal cubriendo un área de $22 \times 45$ metros.

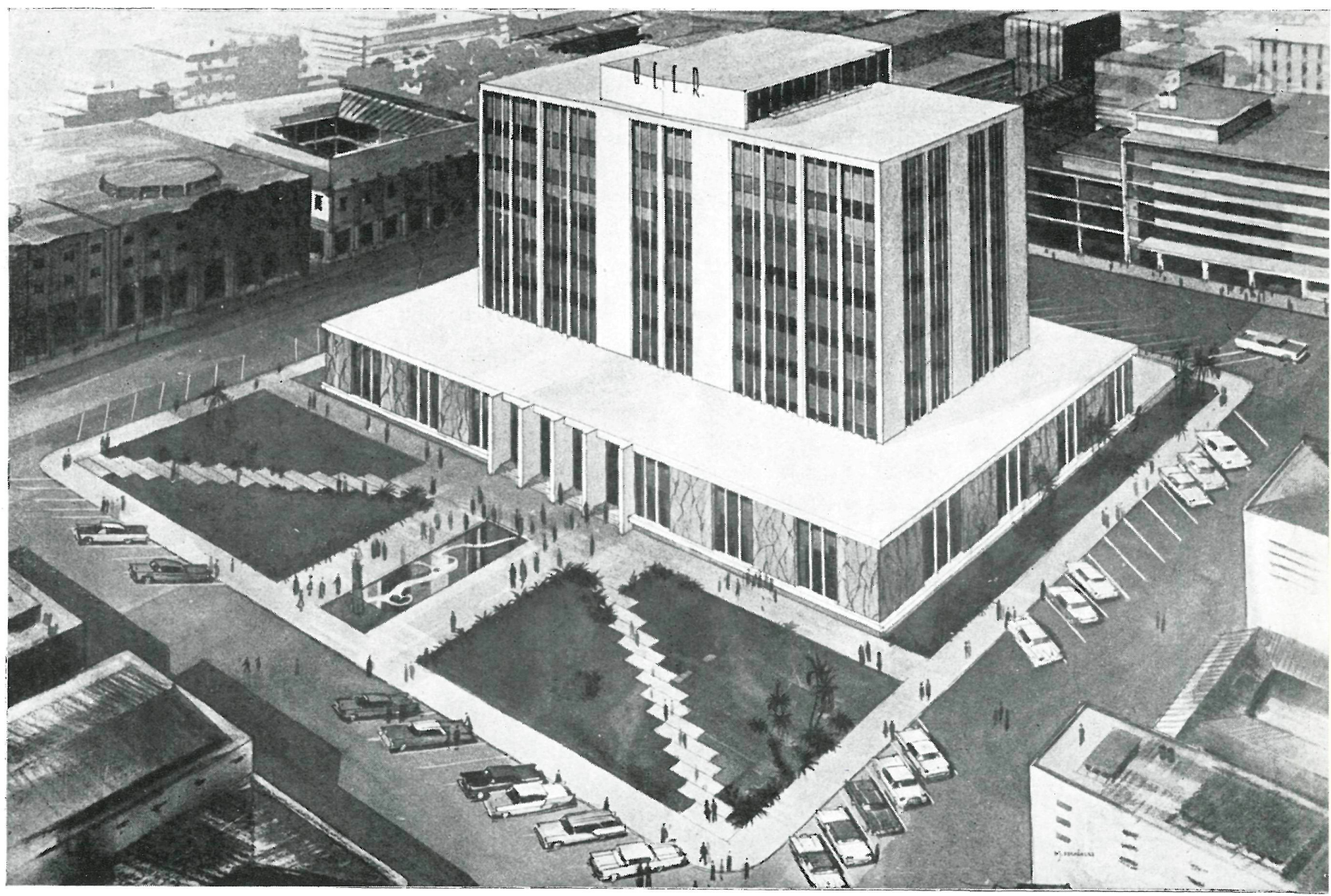




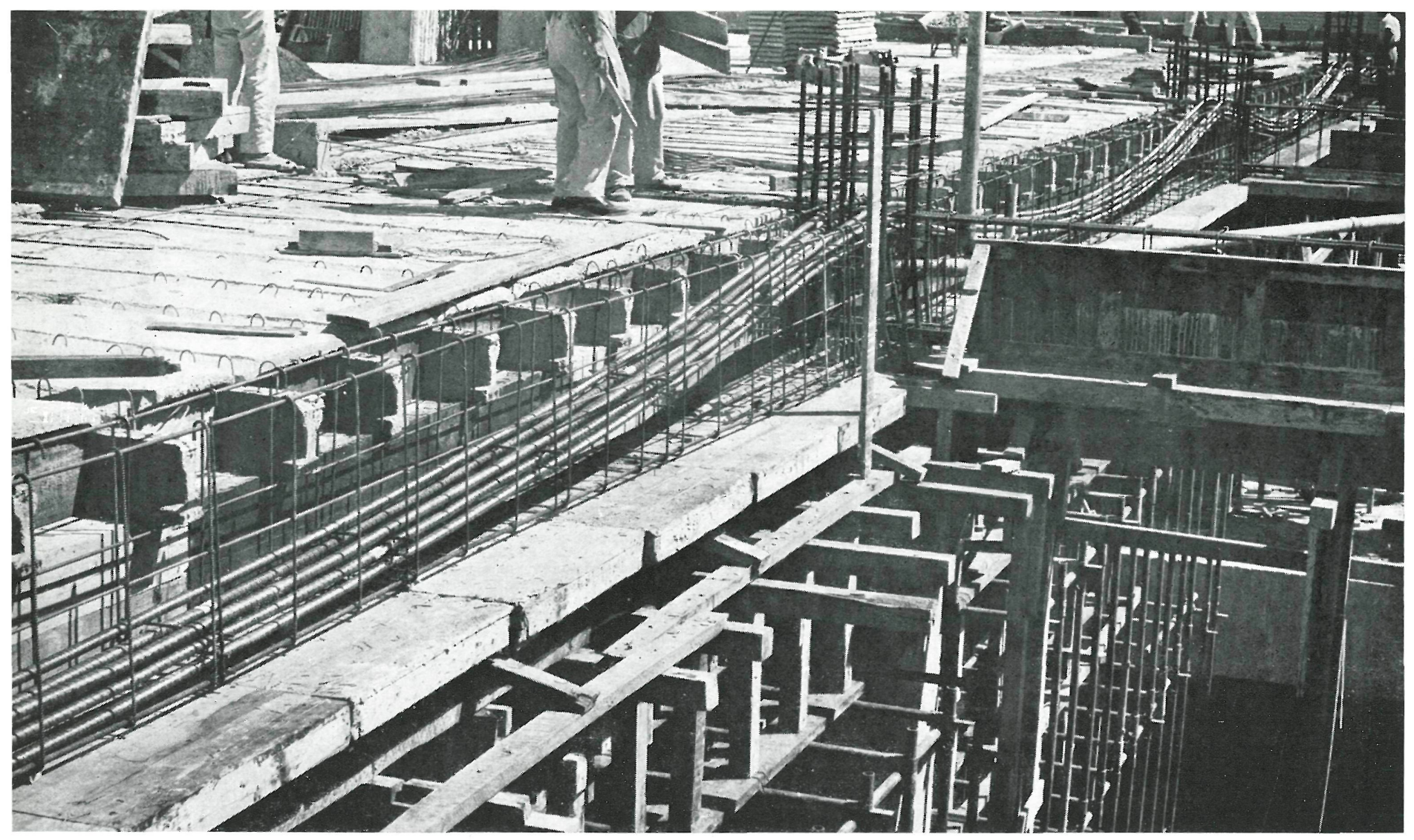

Frente a la entrada principal y sobre la parte de estacionamiento se ha previsto una zona verde (parque) que constribuye al ornato exterior del edificio y permite una vista más amplia del conjunto, dentro de un distrito urbano de calles angostas. La labor del arquitecto refleja un trabajo cuidadoso con miras a obtener un equilibrio justo entre las condiciones estéticas impuestas por el propietario y las tendencias de la arquitectura moderna. Su labor ha sido conjuntar y ponderar las líneas sencillas y materiales de acabado, para imprimir a la obra elegancia y sobriedad.

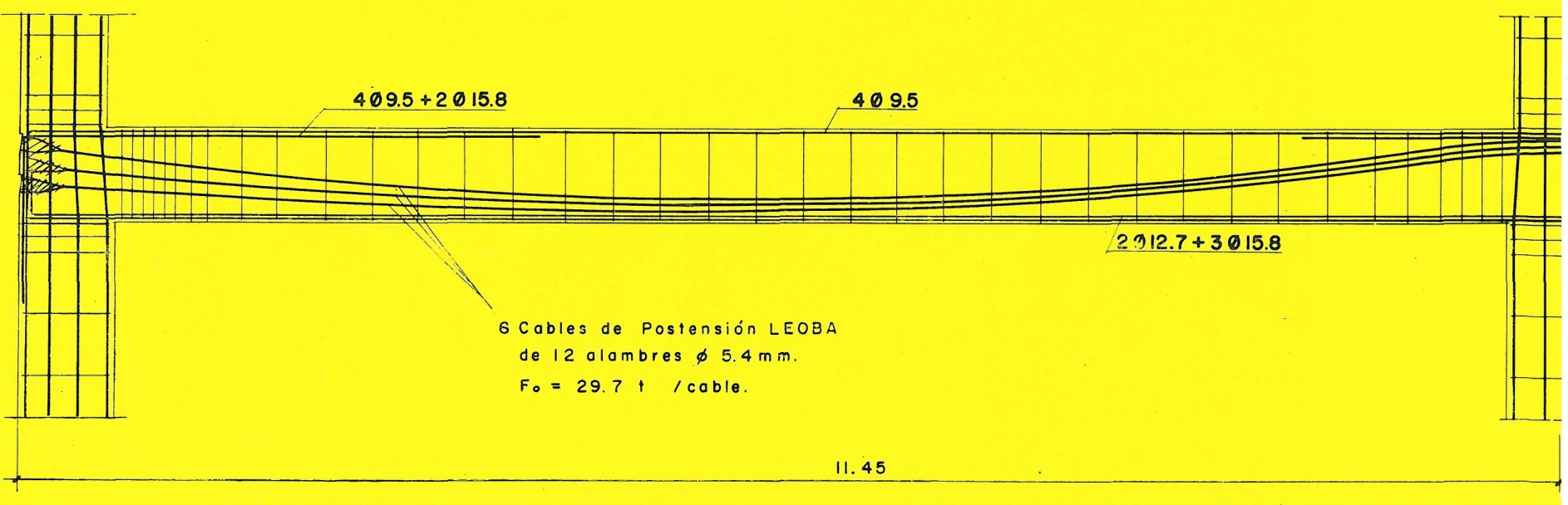

\section{viga postensada}




\section{diagrama de tensiones}

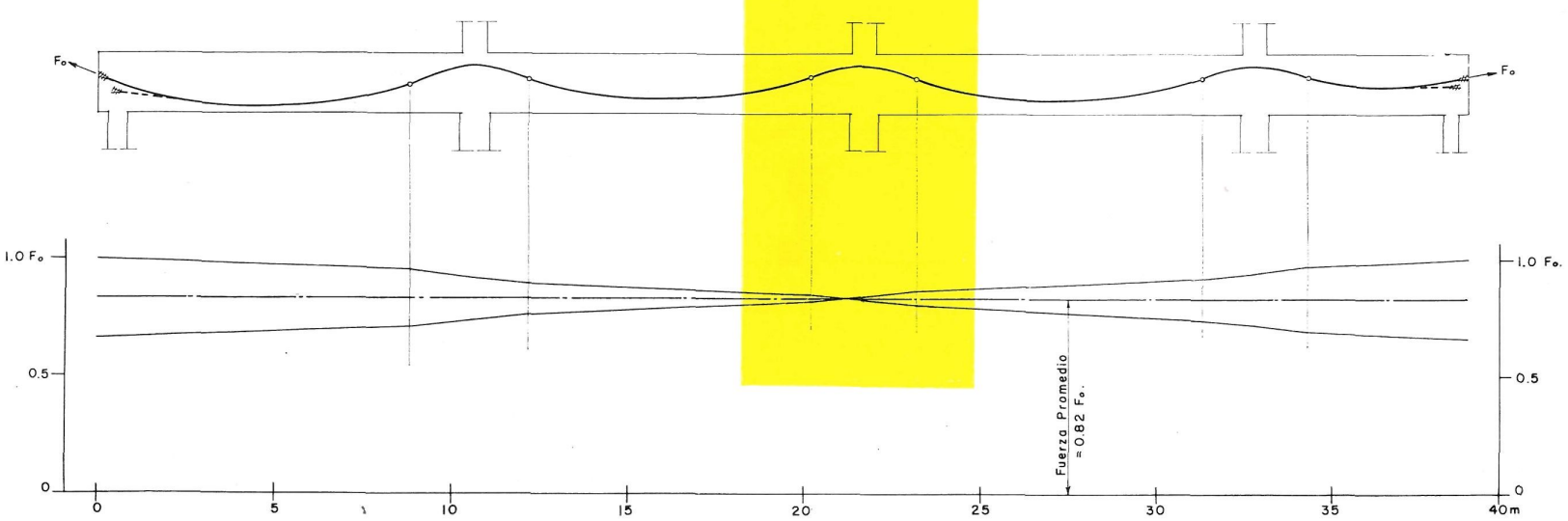

La estructura principal consta de pórticos rígidos formados por pilares de hormigón armado y vigas continuas de hormigón postesado, hormigonados "in situ", y forjados de viguetas pretensadas, prefabricadas en cada planta, en combinación con una losa de forjado, de $5 \mathrm{~cm}$ de espesor, hormigonada simultáneamente con las vigas principales para dar rigidez y monolitismo a la estructura.

Las vigas de carga principales salvan tramos de $11,25 \mathrm{~m}$, y la separación entre ejes de los pórticos es de $7,50 \mathrm{~m}$. Se han obtenido de esta forma distancias modulares entre columnas de $11,25 \times 7,50 \mathrm{~m}$, que permiten un mejor aprovechamiento del espacio útil y dan mayor flexibilidad al planeamiento y distribución funcional del edificio. El empleo del hormigón pretensado ha permitido, a su vez, reducir las dimensiones e influir favorablemente en el comportamiento de los elementos de carga, siendo el resultado una estructura limpia y esbelta, que llena satisfactoriamente las exigencias de la construcción moderna y cumple con los requisitos de economía, sencillez y rapidez de ejecución, y de rigidez y seguridad ante las fuerzas horizontales a que puede estar sometida en una zona de movimientos sísmicos.

En las plantas superiores que constituyen la torre principal del edificio, las vigas postesadas son continuas sobre dos tramos de 11,25 metros cada uno; en las plantas inferiores la continuidad se extiende a cuatro tramos con una longitud total de 39,70 metros. El canto de las vigas, incluyendo el espesor del forjado, es constante y de $70 \mathrm{~cm}$, a excepción de las tres plantas inferiores, en las cuales se aumentó esta dimensión a $73 \mathrm{~cm}$ debido a las mayores solicitaciones de carga móvil y temblor.

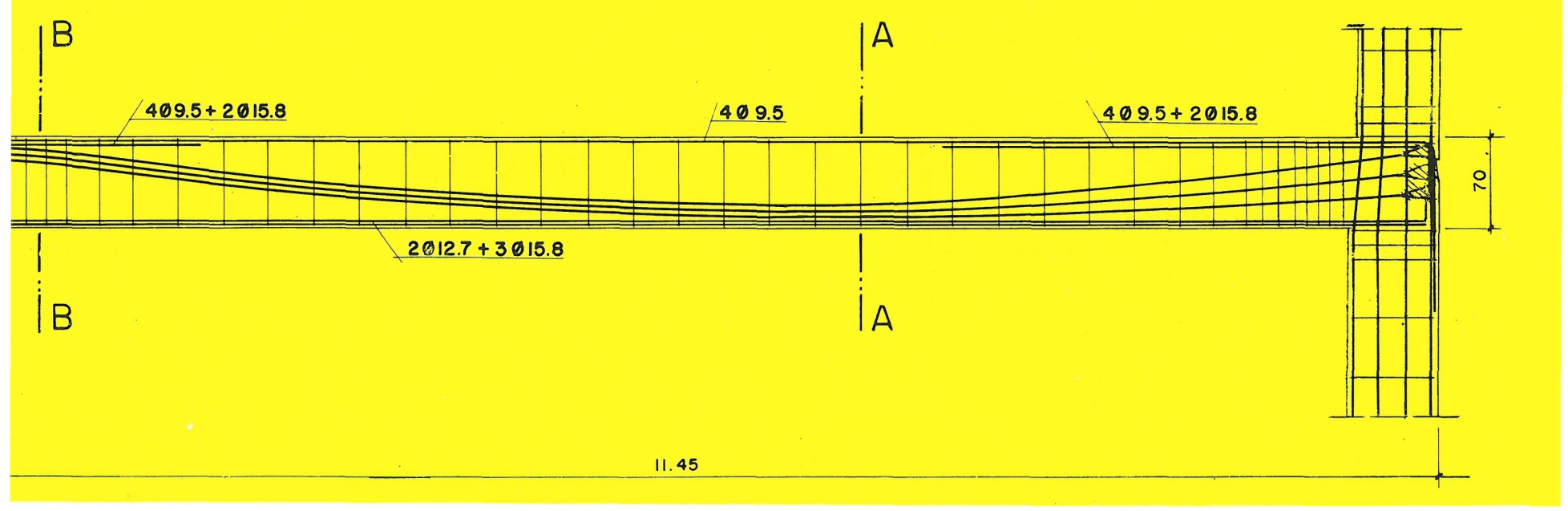

sección longitudinal 

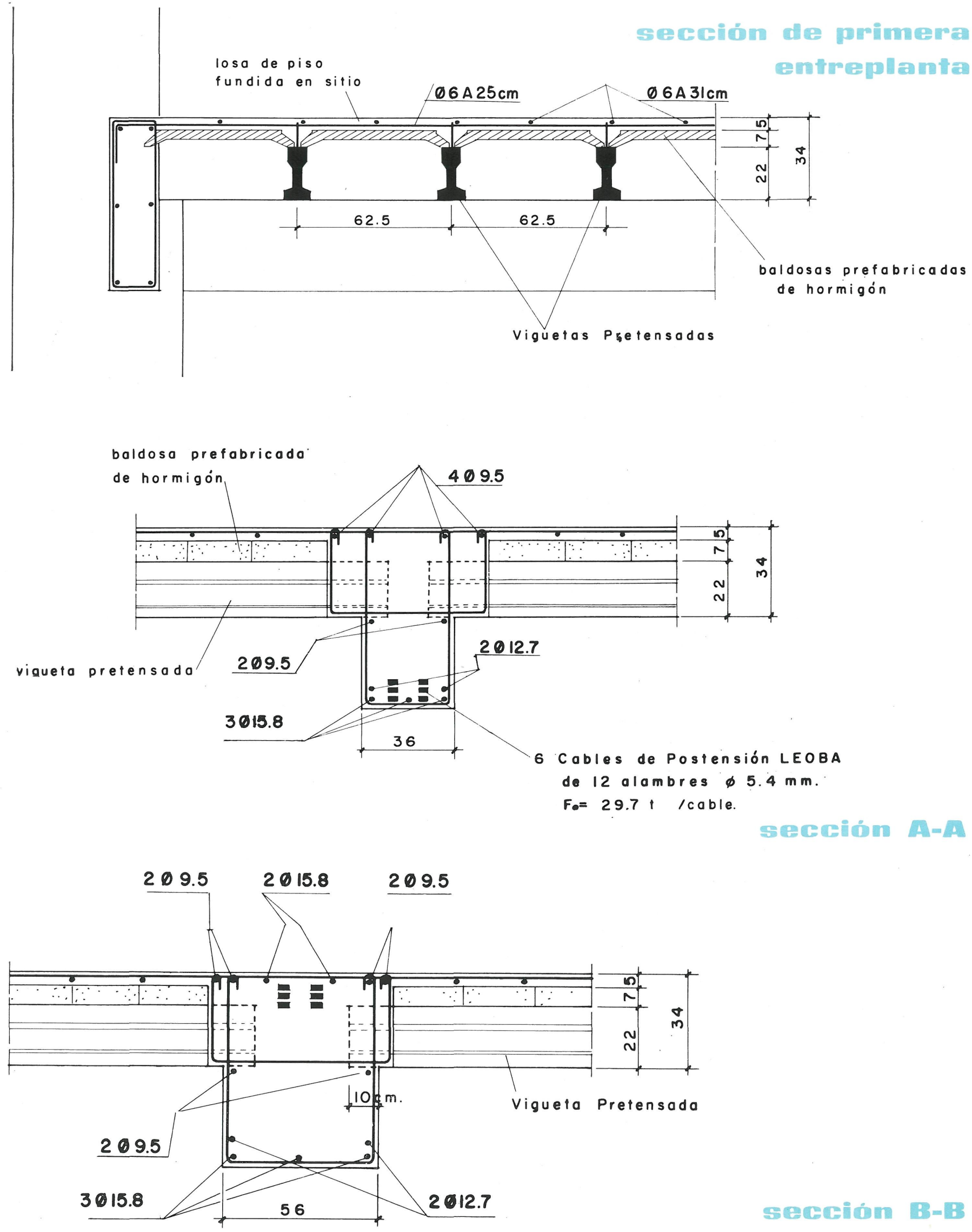

Sección B-B 

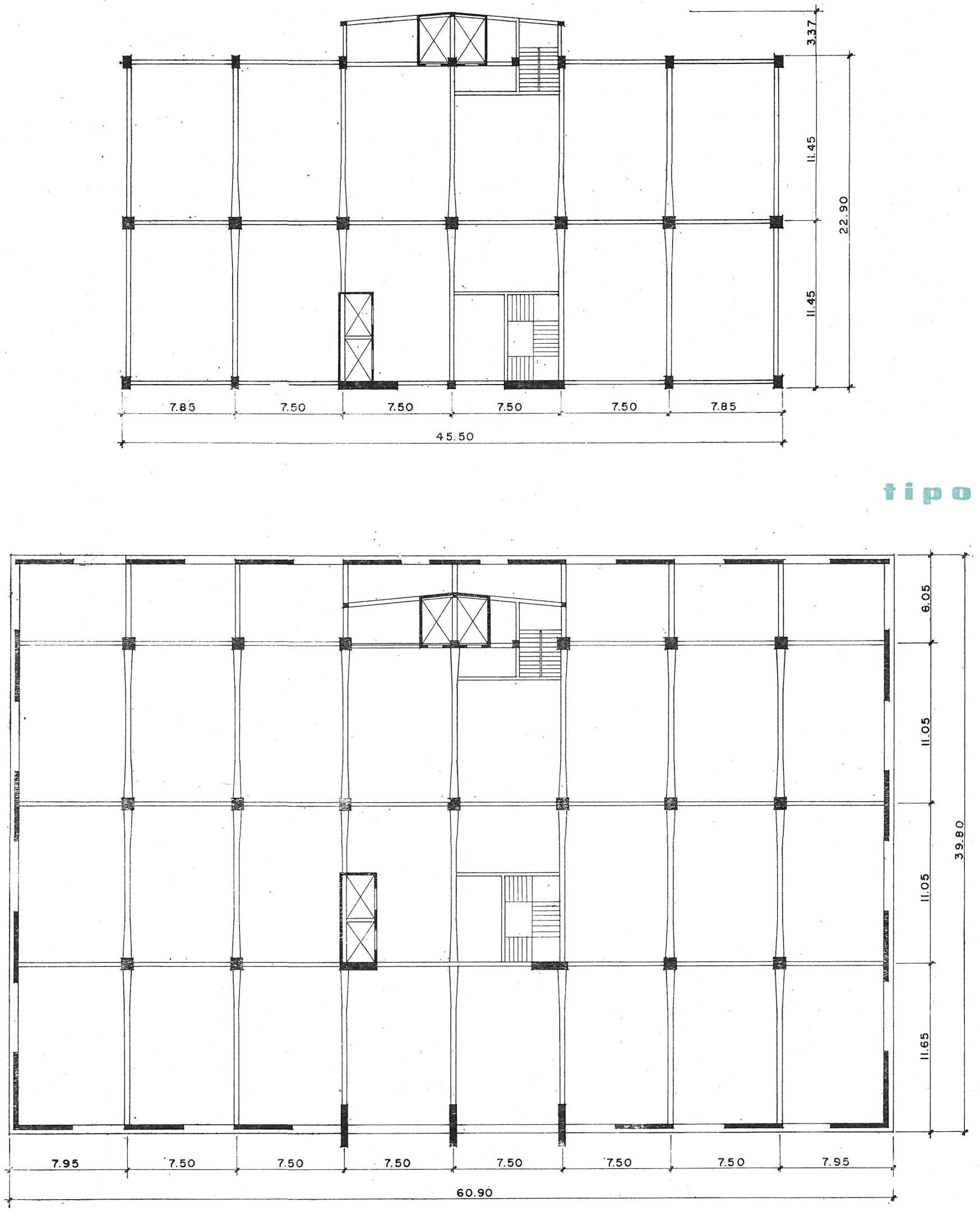
Las vigas tienen un espesor de $36 \mathrm{~cm}$, suficiente para disponer los cables de postesado dejando espacio amplio para colocar y vibrar el hormigón sin dificultad. Sobre los pilares interiores se aumentó esta dimensión gradualmente hasta $56 \mathrm{~cm}$, formando un ensanchamiento horizontal, con objeto de proveer mayor área de compresión en las zonas de momentos de flexión negativos. Las vigas postesadas se hormigonaron integralmente con la losa de forjado de $5 \mathrm{~cm}$ de espesor y actúan con ésta como sección T. Apartándose del criterio seguido por las normas, que especifican el ancho efectivo del ala de vigas $T$ únicamente en función del espesor de la losa, se estimó en los cálculos un ancho efectivo de 3,50 metros basado en la relación de luz de viga a separación entre ejes.

Cada viga fue pretensada por medio de seis cables de postesado-sistema Leoba, original del Dr. Ing. Fritz Leonhardt-continuos sobre varios tramos en toda la longitud de la viga y dispuestos en tres capas horizontales según una línea poligonal parabólica que es afín a la variación de los momentos de flexión en una viga continua para carga uniforme. Los cables de postesado constan, cada uno, de 12 alambres $\varnothing 5,4 \mathrm{~mm}$ de acero ST. $160 / 180$, estirado en frío, con límite de fluencia $(0,2 \%)$ de $160 \mathrm{~kg} / \mathrm{mm}^{2}$, y resistencia de rotura de $180 \mathrm{~kg} / \mathrm{mm}^{2}$. Se tesaron los cables con una tensión admisible de $108 \mathrm{~kg} / \mathrm{mm}^{2}$, siendo la fuerza inicial de pretensado de $29.700 \mathrm{~kg}$ por cable.

Los alambres se colocan en vainas de metal corrugado, de sección ovalada. Su disposición en dos capas horizontales, que se asegura por medio de separadores simples estampados de hojalata, es conveniente para reducir el coeficiente de rozamiento y disminuir las pérdidas de tensión producidas por la curvatura del cable, que para el caso de vigas continuas adquieren especial importancia.

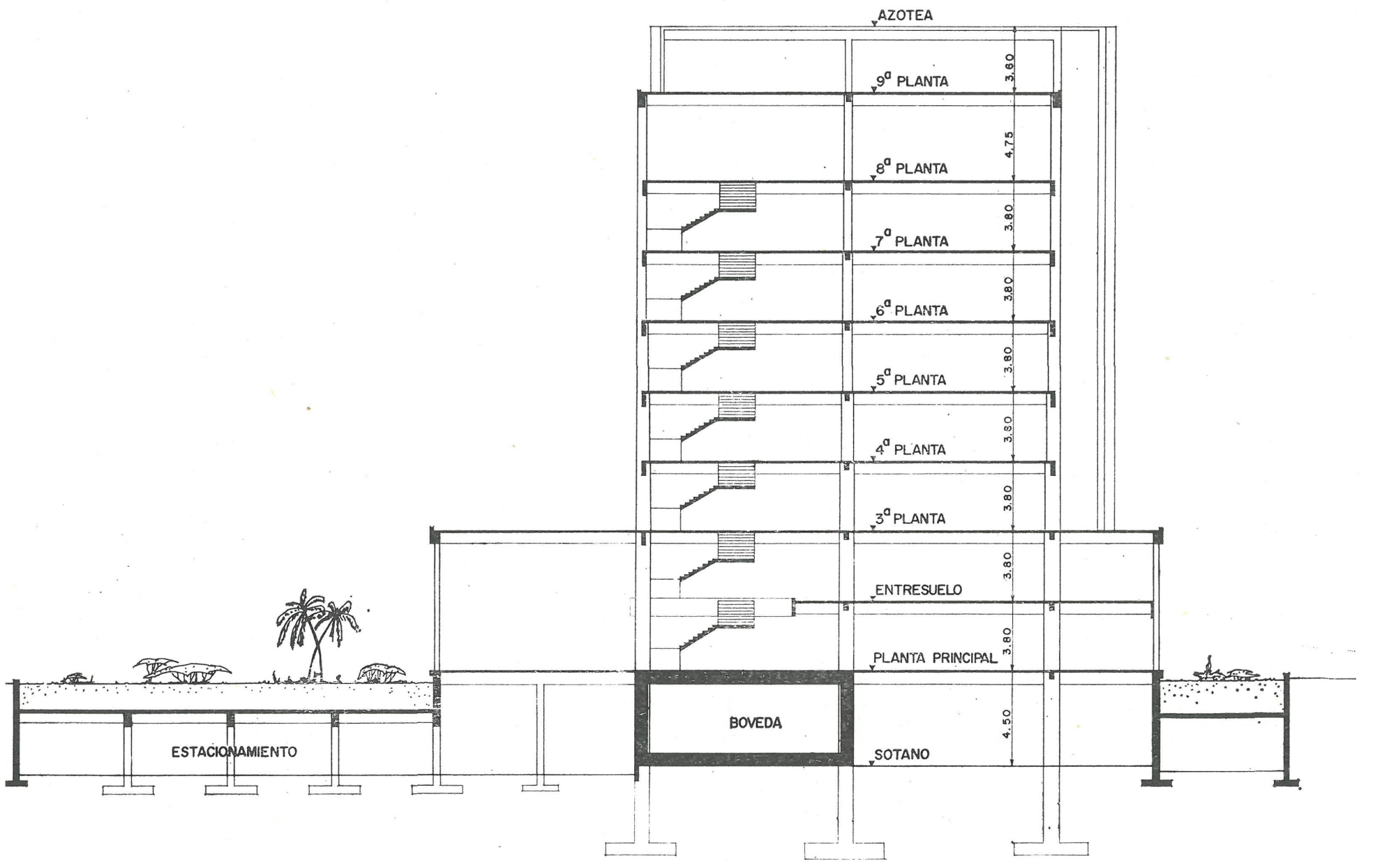

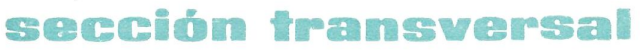


Los cables se tesan únicamente desde un extremo, siendo el otro anclaje fijo por adherencia con el hormigón de la viga. Dichos cables se dispusieron alternando los extremos de tensión, de forma que fueran tesados la mitad de ellos desde cada extremidad de la viga. Se obtiene así una fuerza de pretensado prácticamente constante a lo largo de la viga, y la caída efectiva de tensión por rozamiento se reduce a la mitad de su valor total. El pretensado de vigas continuas y con gran número de tramos es, como lo demuestran los resultados prácticos, factible y económico, y si se realiza con el debido cuidado no presenta dificultades constructivas. Se emplearon vainas de metal corrugado, de gran rigidez, para evitar abolladuras durante su manipulación, y la colocación de los cables se realizó con especial cuidado y exactitud.

En el cálculo de la estructura se consideraron pórticos rígidos de 10 plantas constituidos por pilares de hormigón armado y vigas postesadas. Por estar los cables dispuestos según una curva parabólica fue suficientemente exacto asumir eI efecto de la fuerza de pretensado como una carga equivalente, uniformemente repartida, de sentido contrario a las sobrecargas, más una fuerza de compresión axial. El efecto que la misma produce sobre los elementos del pórtico, vigas y pilares, se analizó de igual forma que la carga permanente, habiéndose empleado el método de aproximaciones sucesivas de Kani para la distribución de los momentos (Referencia: KaniBerechnung Mehrstöckiger Rahmen-Verlag Konrad Wittwer, Stuttgart). Debido a las dimensiones grandes de los pilares en las plantas inferiores y a la oposición que los mismos ofrecen al acortamiento elástico de las vigas producido por el pretensado, fue necesario investigar este efecto y calcular la caída de tensión en los cables y los momentos de flexión inducidos en el pórtico. Como consecuencia del pretensado desaparece en las plantas superiores el efecto de oposición antes citado, que, en general, no resultó determinante para el dimensionado y no afecta fundamentalmente la seguridad a la rotura de la estructura. Se produce además, en el transcurso del tiempo, una redistribución favorable de esfuerzos debida al flujo plástico del hormigón. De las observaciones prácticas se comprobó también que el pretensado de pórticos de este tipo, aun con pilares de dimensiones y rigideces grandes, no presenta dificultad alguna, y que la oposición a las deformaciones elásticas no tiene efecto desfavorable sobre el comportamiento de la estructura.

En el análisis de cargas horizontales, debidas a seísmos no se consideraron como elementos resistentes las paredes laterales de la torre ni la caja del ascensor, por no llegar hasta el nivel de cimientos y ser soportados, en un caso, por pilares independientes y, en el otro, por la losa de la bóveda de seguridad. Por esta razón se analizó cada pórtico actuando independientemente de los otros. El hormigón para los pilares se especificó con una resistencia a los veintiocho días de $\mathbf{3 5 1 , 5 3}$ kilogramos $/ \mathrm{cm}^{2}$ con probetas cilíndricas de $15,2 \times 30,4 \mathrm{~cm}, \mathrm{y}$ para las vigas postesadas, losa de forjado y elementos de carga secundarios de $246,07 \mathrm{~kg}$ /centímetro cuadrado.

Las viguetas pretensadas que forman el forjado son de sección I modificada de $22 \mathrm{~cm}$ de canto, siendo el ancho del ala inferior $11 \mathrm{~cm}$. El refuerzo del pretensado consiste en alambres de acero de sección rectangular, con corrugaciones transversales, para mejorar la adherencia. Las viguetas fue- 
ron prefabricadas en un taller central en bancos de 60 metros. El hormigón empleado en su fabricación fue de $492,15 \mathrm{~kg} / \mathrm{cm}^{2}$ de resistencia.

La separación entre viguetas es de $62,5 \mathrm{~cm}$, y sobre su parte superior se apoyaron baldosas delgadas de hormigón, prefabricadas, que sirven de encofrado para hormigonar la losa de forjado de $5 \mathrm{~cm}$. El espesor total del forjado es de $34 \mathrm{~cm}$, e incluye vigueta, baldosa prefabricada y losa del forjado. La conexión entre viguetas pretensadas y losa, y su comportamiento como sección compuesta se asegura por medio de adherencia y estribos de $\varnothing 6,35 \mathrm{~mm}$ que sobresalen de las viguetas. En el centro del tramo se dispuso un tabique diafragma transversal hormigonado "in situ" que une las viguetas con objeto de distribuir posibles concentraciones de cargas.

El proceso de construcción se inició en cada planta con la preparación de los encofrados de madera y con la colocación del refuerzo de acero y cables de postesado de las vigas principales. Las viguetas prefabricadas se soportaron directamente sobre el encofrado penetrando en la viga aproximadamente $12 \mathrm{~cm}, \mathrm{y}$ quedando de esta forma embebidas en el hormigón. Se procedió luego a la colocación de las baldosas prefabricadas del forjado, del refuerzo de acero de la losa y de las vigas secundarias. La losa del forjado, vigas principales y elementos de carga secundarios se hormigonaron simultáneamente, siencio el resultado un sistema de forjado monolítico y de gran rigidez para resistir cargas sísmicas horizontales. La losa de hormigón, fabricada "in situ" cumple simultáneamente varias funciones: sirve como superficie de solado, y en combinación con las viguetas pretensadas forma parte resistente de la entreplanta; asimismo actúa como ala de compresión de las vigas postesadas y, como se mencionó antes, tiene como función principal la unión integral de los elementos prefabricados con la estructura para asegurar la rigidez necesaria.

Las viguetas se apoyaron provisionalmente en el centro con montantes tubulares antes de proceder a la colocación de las baldosas. De esta forma el peso propio del forjado y la sobrecarga son soportados por la sección compuesta constituida por viguetas y losa del forjado hormigonada "in situ". Se aprovechó la continuidad del forjado disponiendo sobre las vigas principales barras de refuerzo convencional que absorben tensión en las zonas de momentos negativos. En estas zonas resultan críticos los esfuerzos de compresión en el ala inferior de las viguetas por presentar éstas una zona de compresión reducida, obteniéndose esfuerzos que sobrepasan para el estado de sobrecarga total los valores admisibles. Se siguió, por lo tanto, un criterio distinto en el diseño. Se dimensionó la sección del apoyo de tal forma que fuese capaz de resistir los momentos producidos en el estado de carga permanente más el $50 \%$ de la sobrecarga sin sobrepasar los esfuerzos admisibles en el acero y hormigón. Para el estado de sobrecarga total, estos esfuerzos sobrepasan los admisibles en un $30 \%$, manteniéndose, sin embargo, suficiente seguridad al agrietamiento. El estado de rotura se basó en el momento máximo de las secciones de apoyo, después del cual estas secciones se comportan como articulaciones plásticas, produciéndose una redistribución de momentos y siendo absorbido el exceso de carga por la sección del centro del tramo. La seguridad a la rotura resultaba satisfactoria. 


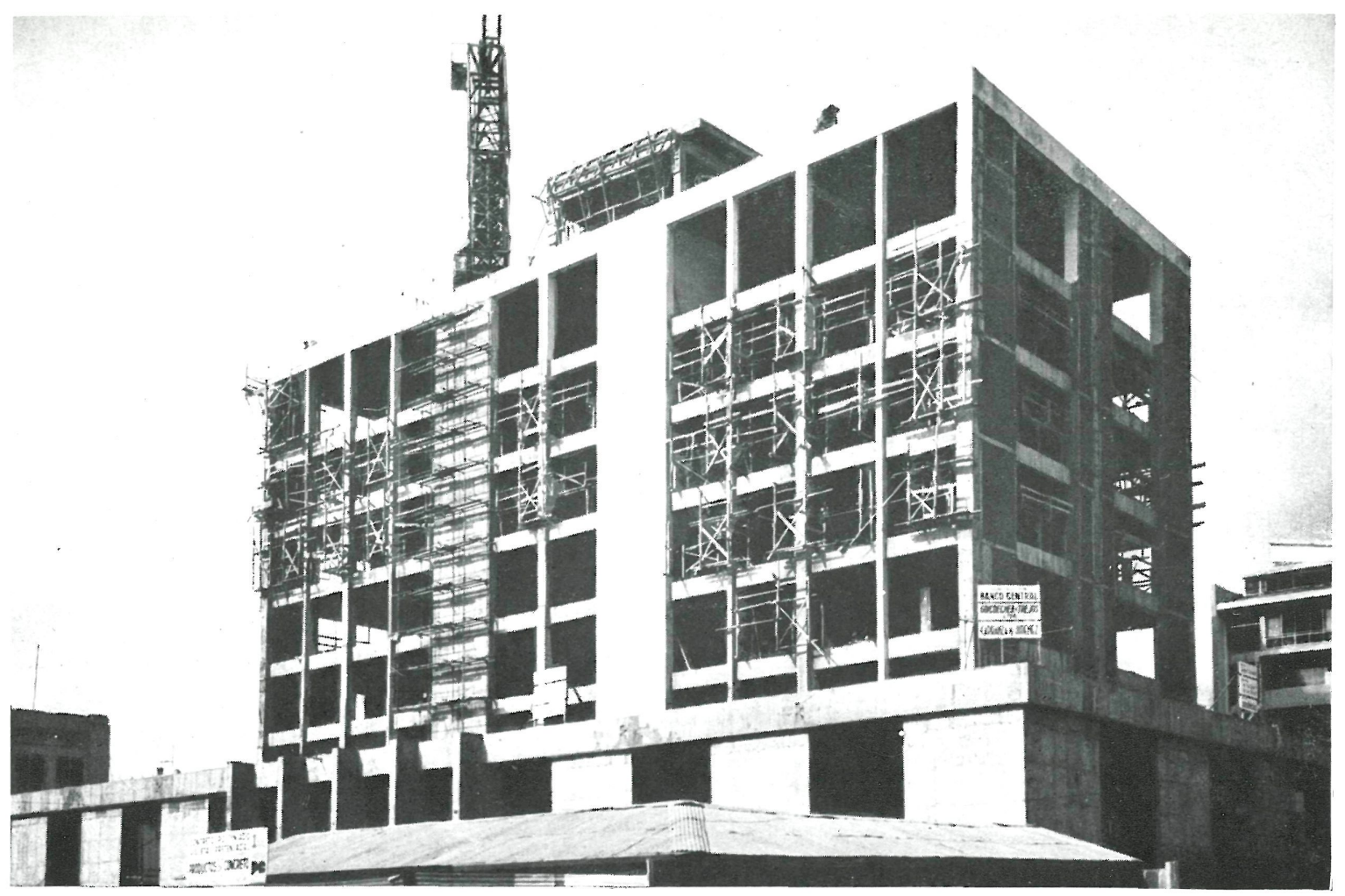

Solamente se requirieron encofrados de madera en las vigas postesadas y pilares, así como para los elementos secundarios.

El arquitecto Jorge Escalante Van Patten tuvo bajo su responsabilidad el trabajo arquitectónico de la obra. El proyecto de la estructura fue realizado por el autor en colaboración con el ingeniero Eddy Bravo. Los contratistas generales de la obra fueron las empresas constructoras Carranza \& Jiménez Ltda. y Goicoechea \& Trejos Ltda. Productos de Concreto, S. A., ejecutó todo el trabajo de colocación, tesado e inyección de los cables de postesado y subcontrató la fabricación de las viguetas pretensadas para los forjados. La construcción se inició a principios del año 1961, y se espera concluir la obra a principio de 1963.

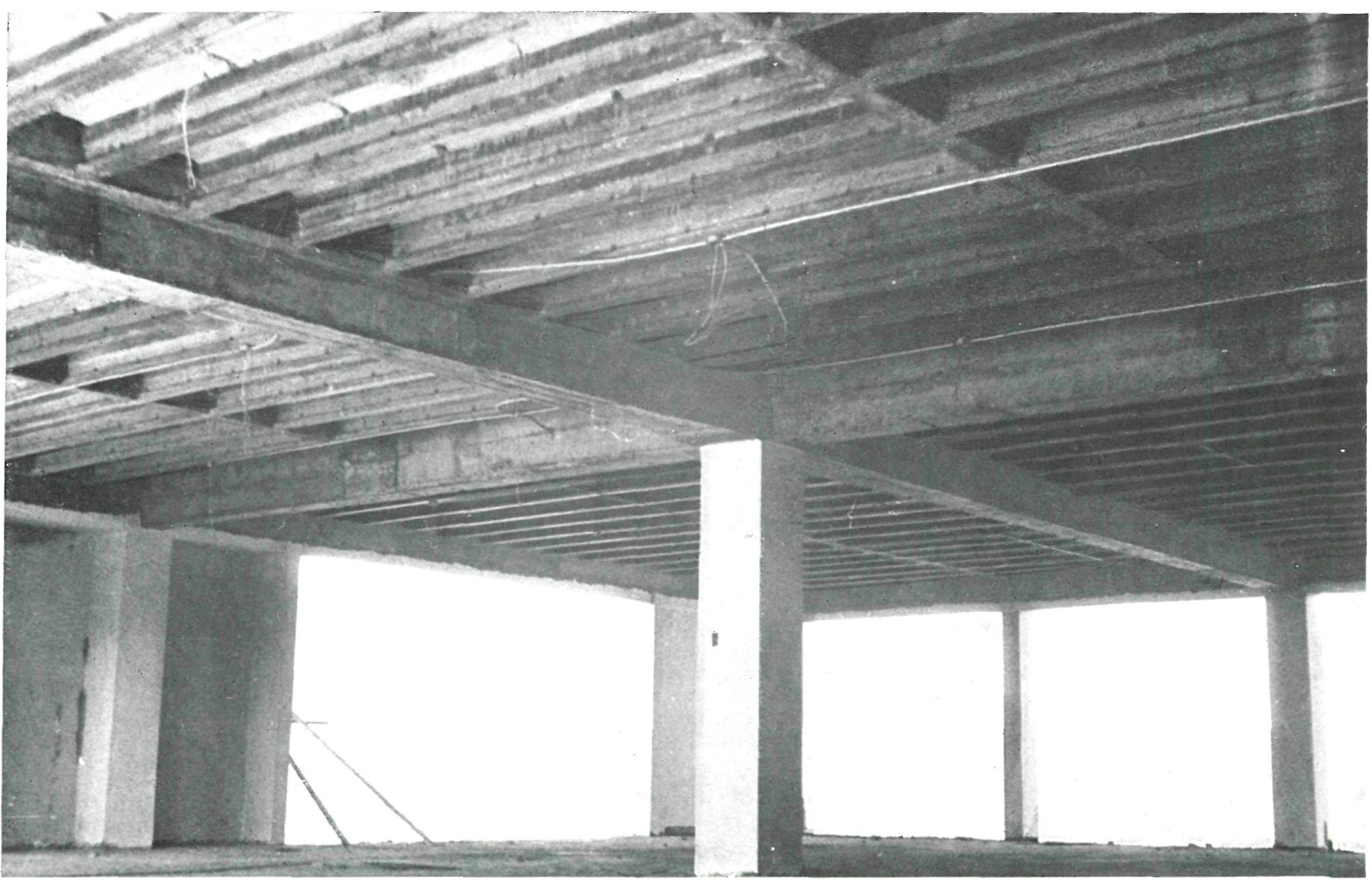




\section{mó}

\section{Banque Centrate du Costa Rica}

Franz Sauter, ingénieur civil.

Le nouvel édifice destiné à grouper les services de la Banque Centrale du Costa Rica est composé d'une structure en béton précontraint. L'immeuble dispose d'un sous-sol, d'un propre stationnement pour 105 véhicu. les et compte neuf étages. Sa superficie totale est de 12.000 mètres carrés.

Il repose sur une base de 40 mètres sur 60, d'où s'élève un ensemble central de 22 mètres sur 45.

Bien que situé dans un quartier de rues étroites, la façade principale donne sur une zone où un parc est prévu, ce qui procurera à l'édifice, avec une meilleure visibillité, une catégorie plus élevée.

La structure est composée d'un treillis de poutres précontraintes et de piliers qui ont été bétonnés en chantier. Les poutrelles, également précontraintes, ont été préfabriquées en atelier. Le treillis formé à chaque étage par ces éléments résistants constitue l'armature d'une dalle. Cette forme monolithe assure une excellente étage par ces éléments
rigidité à la structure.

Les poutres principales, continues, franchissent des tronecons de $11,25 \mathrm{~m}$ de portée, avec des sections variées et les armatures de précontrainte sont de 6 câbles type Leoba, original du Dr. Ing. Fritz Leonhardt, disposés et les armatures de précontrainte sont de 6 câbles type Leoba, original du Dr. Ing. Fritz Leonhardt, disposés en trois couches horizontales et présentant un profil parabolique. Chacun des câbles comporte 12 fils, de $5,4 \mathrm{~mm}$
de diamètre et d'une résistance de $180 \mathrm{~kg} / \mathrm{mm}^{2}$ à la rupture. La tension était de $108 \mathrm{~kg} / \mathrm{mm}^{2}$ et l'effort total de de diamètre et d'une résistance de $180 \mathrm{~kg} / \mathrm{mm}^{2}$ à la rupture. La tension était de $108 \mathrm{~kg} / \mathrm{mm}^{2}$ et l'effort total de
précontrainte par câble de $29.700 \mathrm{~kg}$. Les câbles ont été placés à l'intérieur de gaines de métal crénelé dont les espacements ont été maintenus à l'aide d'éléments métalliques.

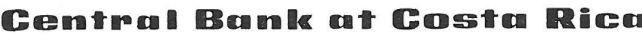

Franz Sauter, civil engineer.

This new building is intended to house the various services of the Central Bank of Costa Rica. It has a prestressed concrete structure, and consists of a basement parking space for 105 vehicles, and nine storeys, providing altogether a floor surface of $12,000 \mathrm{~ms}^{2}$.

The building rests on a ground area of 40 by $60 \mathrm{~ms}$, and the main structure occupies 22 by $45 \mathrm{~ms}$.

This Bank is located in a district of narrow streets, but its main side overlooks a green ojen space, which will improve its visibility and appearance.

The building structure is made up of a framework of prestressed beams and columns. The beams have been The building structure is made up of a framework of prestressed beams and colum This fraricework, at each concreted at the site, and the joists, which are also prestressed, are factory made. This frainework, at each
floor level, constitutes the basis of a continuous slab, which renders the total structure exceedingly stiff.

The main continuous girders span $11.22 \mathrm{~ms}$ spaces, and vary in cross section. The prestressing reinforcements consist of 6 Loeba type cables. This is an original design by Dr. Leonhardt, in which the cables are placed on three horizontal layers, of parabolic outline. Each cable is made up of $12 \times 5.4 \mathrm{~mm}$ wires, with a breaking stress of $180 \mathrm{~kg} / \mathrm{mm}^{2}$. The tensioning stress was $108 \mathrm{~kg} / \mathrm{mm}^{2}$, and the total prestress load is $29,700 \mathrm{kgs}$. The cables run in corrugated metal tubes, and these were kept in precise position with the aid of distance pieces.

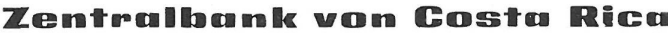

Franz Sauter, Bauingenieur.

Das neue Gebäude, das dazu bestimmt ist, die Diensträume der Zentralbank von Costa Rica aufzunehmen, wird von einer Spannbeton-Struktur gebildet. Das Haus verfügt über einen Keller, eine eigene Parkanlange für 105 Fahrzeuge und neun Stockwerke, mit einer Gesamtoberfläche von $12.000 \mathrm{~m}^{2}$

Es setzt sich auf einen Grund von $40 \times 60 \mathrm{~m}$, wo sich ein Zentralkern von $22 \times 45 \mathrm{~m}$ erhebt.

Es liegt in einem Viertel mit engen Strassen, jedoch geht seine Hauptfassade auf eine Grünanlage, die ihm einen höheren Rang und bessere Sichtbarkeit verleiht.

Die Struktur setzt sich aus einem Gitterliniennetz aus Spannbalken und Pfosten zusammen. Die Balken wurden beim Bau betoniert und die kleinen Balken, ebenfalls gespannt, in der Werkstatt hergestellt. Das Gitterliniennetz, das die genannten widerständigen Elemente in jedem Geschoss bilden, macht das Gestell für eine Platte aus. Diese monolithische Form verleiht der Struktur grosse Steifigkeit.

Die durchlaufenden Hauptbalken überspannen Abschnitte von 11,25 m Weite mit mehreren Schnitten. Die Die durchlaufenden Hauptbalken uberspannen Abschnitte von 11, $\mathrm{m}$ Weite mit mehreren Schnitten. Die Spannbewehrungen bestehen aus 6 Kabeln nach Leoba, einem Original des Dr. Ing. Fritz Leonhardt, die in
drei horizontalen Schichten angelegt wurden und ein parabolisches Profil aufweisen. Jedes der Kabel setzt
sich aus 12 Drähten von 5,4 mm Durchmesser zusammen, mit einer Bruchfestigkeit von 180 $\mathrm{kg} / \mathrm{mm}^{2}$. Die Spannung des Vorspannens betrug $108 \mathrm{~kg} / \mathrm{mm}^{2}$ und die Gesamtkraft der Vorspannung $29 . \% 00 \mathrm{~kg}$ je Kabel. Die Kabel wurden in das Innere von gewellten Metallhülsen hineingelegt, welche ihrerseits durch metallische Abstandshalter in ihrer Stellung erhalten werden. 